\title{
Intensity modulated radiotherapy for sinonasal malignancies with a focus on optic pathway preservation
}

\author{
Alexander Chi ${ }^{{ }^{*}}$, Nam P Nguyen ${ }^{2}$, William Tse ${ }^{3}$, Gill Sobremonte ${ }^{4}$, Patrick Concannon ${ }^{4}$ and Angela Zhu ${ }^{4}$
}

\begin{abstract}
Purpose: To assess if intensity-modulated radiotherapy (IMRT) can possibly lead to improved local control and lower incidence of vision impairment/blindness in comparison to non-IMRT techniques when treating sinonasal malignancies; what is the most optimal dose constraints for the optic pathway; and the impact of different IMRT strategies on optic pathway sparing in this setting.

Methods and materials: A literature search in the PubMed databases was conducted in July, 2012.

Results: Clinical studies on IMRT and 2D/3D (2 dimensional/3 dimensional) RT for sinonasal malignancies suggest improved local control and lower incidence of severe vision impairment with IMRT in comparison to non-IMRT techniques. As observed in the non-IMRT studies, blindness due to disease progression may occur despite a lack of severe toxicity possibly due to the difficulty of controlling locally very advanced disease with a dose $\leq 70 \mathrm{~Gy}$. Concurrent chemotherapy's influence on the the risk of severe optic toxicity after radiotherapy is unclear. A maximum dose of $\leq 54$ Gy with conventional fractionation to the optic pathway may decrease the risk of blindness. Increased magnitude of intensity modulation through increasing the number of segments, beams, and using a combination of coplanar and non-coplanar arrangements may help increase dose conformality and optic pathway sparing when IMRT is used.
\end{abstract}

Conclusion: IMRT optimized with appropriate strategies may be the treatment of choice for the most optimal local control and optic pathway sparing when treating sinonasal malignancy.

Keywords: Intensity modulated radiotherapy, Sino-nasal malignancies, Optic toxicity, Blindness

\section{Background}

Being less than $1 \%$ of all cancer, sinonasal malignancies frequently present in the locally advanced stage [1,2]. This poses a therapeutic challenge in the treatment planning and delivery of definitive or adjuvant radiotherapy due to the proximity of the primary tumors to critical normal structures, such as the optic nerves $(\mathrm{ON})$ and chiasm (OC). Severe vision impairment and blindness are often encountered after therapeutic doses were delivered in the pre-IMRT era [3-19]. This was most commonly observed in the era of conventional radiotherapy (2D), when doses to the target volume and adjacent

\footnotetext{
* Correspondence: achiaz2010@gmail.com

'Department of Radiation Oncology, West Virginia University, 1 Medical

Center Dr. Morgantown, Morgantown, WV 26506, USA

Full list of author information is available at the end of the article
}

critical structures could not be well defined [3-9,14-19]. Due to the lack of the ability to deliver a highly conformal radiation dose, the clinical outcome in the $2 \mathrm{D}$ era is also poor [3-9]. As an ideal approach to deliver a meaningful dose to the primary tumor or the postoperative tumor bed, and optimally preserve the optic pathway, which is often immediately adjacent to the planning target volume (PTV), intensity modulated radiotherapy (IMRT) has been utilized with excellent short term results $[20,21]$. Since the adaptation of IMRT into clinical practice, the clinical outcome of IMRT for sinonasal malignancies have been reported by many institutions with improved visual preservation [22-26]. However, there was no consensus on the dose limits for optimal visual preservation. Thus, we conduct this review to analyze if the incidence of vision impairment and

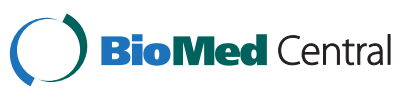


blindness after IMRT is less frequent in comparison to conventional/three dimensional conformal radiotherapy (3D-CRT) when treating sinonasal malignancies and local tumor control follow IMRT when compared with non-IMRT techniques; the relationship of vision impairment and radiation dose to the optic pathway in this setting; and how various strategies of IMRT delivery may influence optic structure sparing.

\section{Methods and materials}

This systematic review of literature was to investigate the clinical outcome and the incidence of vision impairment and blindness relative to the doses to the optic pathway after 2D RT, 3D-CRT, and IMRT for sinonasal malignancies in order to demonstrate if IMRT can potentially lower the incidence of vision impairment without any compromise of tumor control. In addition, we will explore the potential advantages \& disadvantages of various IMRT delivery strategies for optic pathway sparing in this setting. A search based on PubMed electronic databases was conducted in July, 2012 to select studies outlining the following: clinical outcome of radiotherapy for sinonasal or paranasal malignancies; vision impairment and blindness after radiotherapy. The following terms were explored and used for each database search: sinonasal malignancies, paranasal sinus tumors, nasal cavity \& ethmoid sinus tumors, vision impairment, optic neuropathy, optic retinopathy, blindness, radiotherapy, IMRT. For clinical reports, only the most complete and most recently published full length study reporting the clinical outcome and treatment related toxicity after 2D/3D RT, IMRT, or any combination of them for predominantly carcinomas from the sinonasal complex has been selected from any one institution. Two separate studies from one single institution were selected only if they described the outcome after RT delivered with two different techniques, or when one is reporting the outcome for exclusively one RT delivery approach and another is a comparison study of clinical outcome after RT delivered with various techniques. Studies describing the technical aspects of RT delivery were selected as long as the same investigation was not duplicated by the same group of authors. Conventional fractionation of 1.8 to 2 Gy per day is assumed unless otherwise indicated in this study.

\section{Results}

Fifteen studies published between 1983 and 2009 [3-17], describing the clinical outcome and optic toxicity in detail after 2D or 3D RT; 2 studies published between 2008 \& $2009[18,19]$, reporting the clinical outcome \& optic toxicity profile after RT delivered with a combination of 2D/3D RT \& IMRT; 5 studies published between 2006 and 2012 [22-26], reporting the clinical outcome \& optic toxicity after exclusively IMRT; and 3 studies published between 2007 and 2012 comparing the clinical outcome \& incidence of optic toxicity after IMRT and non-IMRT techniques [27-29] were selected for the clinical portion of this review. Twelve studies describing the technical aspects of IMRT for paranasal malignancies were also selected for exploration of what would be the best approach to optimize optic pathway sparing [30-41].

\section{Local control \& optic toxicity when IMRT is not used, or used in only a portion of patients}

As shown in Table 1, treatment related blindness has been reported in all but one study which delivered a median dose of 54 Gy in 27 daily fractions when only 2D RT was delivered [3-9]. The local control from studies which reported them was $45.4 \%-62 \%$ (Table 1). The lowest local control was associated with a relatively high percentage of T4 disease and lower dose delivered with a hypofractionated course [6].

The local control reported in the four 3D studies was $56.4 \%-83 \%$, while the percentage of locally advanced disease appear to be high when compared with that reported in the 2D studies [10-13] (Tables 1 and 2). The incidence of treatment related blindness appears to be lower in these studies. Only 1 such case was found 3 years after radiotherapy [12]. However, $41.2 \%$ patients experienced severe optic toxicity at 2 years after being treated with concurrently intra-arterial cisplatin and radiotherapy in one study [10]. This study also reported the best local control of $83 \%$ after a median follow up of 55.2 months.

Severe vision impairment/blindness became more prevalent in studies that included patients who received 2D RT and those who were treated with 3D and/or IMRT [14-19] (Table 3). The local control in these studies was $35.9 \%-64.4 \%$. The lowest local control (35.9\%) was found in a study which only included patients with unresectable T4b disease treated to 70 Gy [18]. Otherwise, the local control was $58.5 \%-64.4 \%$ in these studies. It was $62 \%$ in the study which observed blindness due to disease progression only [16]. 56.67\% of the patients had T4 disease on that study. A study that reported both 2D \& 3D results demonstrated the actuarial rate of severe ophthalmological complications at 5 years to be $15 \%$ after 2D RT, but only $4 \%$ after 3D CRT even though this did not reach any statistical significance [15]. In the 2 studies which included 2D/3D RT, and IMRT, blindness is found 7 years after IMRT in one study when an area of the optic nerve received $77 \mathrm{~Gy}$; and mostly after a definitive dose of $70 \mathrm{~Gy} / 35$ daily fractions was delivered in the other study $[18,19]$.

As shown in several studies, the incidence of vision impairment appears to correlate with the radiation dose 
Table 1 Optic toxicity after 2D RT for sinonasal malignancies

\begin{tabular}{|c|c|c|c|c|c|c|c|c|c|}
\hline Ref. & $\#$ & $\begin{array}{l}\text { Median follow } \\
\text { up }(\mathrm{mo})\end{array}$ & Stage & RT & Presciption dose & Local control & $\begin{array}{l}\text { Dose to the } \\
\text { optic pathway }\end{array}$ & $\begin{array}{l}\text { Vision } \\
\text { impairment }\end{array}$ & Blindness \\
\hline $\begin{array}{l}\text { Ogawa } \\
{[3]^{\dagger \S}}\end{array}$ & 41 & 93 & $\begin{array}{l}\mathrm{T}_{4}: \\
31.7 \%\end{array}$ & $2 D$ & $\begin{array}{l}54 \mathrm{~Gy} / 27 \mathrm{frx}{ }^{\neq} \\
(40-70 \mathrm{~Gy})\end{array}$ & $59 \%$ & $54 \& 60 \mathrm{~Gy}^{*}$ & $7.3 \%$ & None \\
\hline $\begin{array}{l}\text { Tiwari } \\
{[4]^{+}}\end{array}$ & 50 & $\mathrm{n} / \mathrm{a}$ & $\begin{array}{l}\text { IV: } \\
50 \%\end{array}$ & $2 \mathrm{D}$ & $\begin{array}{l}64-70 \\
\text { Gy/32-35 frx } \pm \\
\text { brachytherapy } \\
\text { to } 82 \text { Gy }\end{array}$ & $\begin{array}{l}62 \% ; 16 / 19 \\
\text { recurrences are } \\
\text { stage III or IV }\end{array}$ & $\mathrm{n} / \mathrm{a}$ & $\begin{array}{l}16 \% \text { optic } \\
\text { retinopathy } \\
\& \text { neuropathy }\end{array}$ & $2 \%$ unilateral blindness \\
\hline \multirow[t]{3}{*}{$\begin{array}{l}\text { Jiang } \\
{[5]^{\S}}\end{array}$} & \multirow[t]{3}{*}{219} & \multirow[t]{3}{*}{$\mathrm{n} / \mathrm{a}$} & \multirow[t]{3}{*}{$\mathrm{n} / \mathrm{a}$} & \multirow[t]{3}{*}{$2 \mathrm{D}$} & \multirow[t]{3}{*}{$\mathrm{n} / \mathrm{a}$} & \multirow[t]{3}{*}{$\mathrm{n} / \mathrm{a}$} & $\begin{array}{l}\text { Retina: } 58.3 \\
\text { Gy/29 frx }\end{array}$ & Ipsilateral: 33.7\%. & $\begin{array}{l}8.2 \% \text { ipsilateral blindness } \\
\text { due to optic neuropathy; } \\
\text { bilateral blindness due to } \\
\text { chiasm injury at } 10 \text { yrs: }\end{array}$ \\
\hline & & & & & & & $\begin{array}{l}\text { ON: } 61.6 \\
\text { Gy/30 frx; }\end{array}$ & $\begin{array}{l}\text { Retinopathy: 20\% } \\
\text { when 50-60 Gy } \\
\text { received; }\end{array}$ & 50-60 Gy: 8\%; \\
\hline & & & & & & & $\begin{array}{l}\text { OC: } 57.1 \\
\text { Gy/30 frx }\end{array}$ & $\begin{array}{l}\text { Optic neuropathy: } \\
2.3 \% \text { when optic } \\
\text { nerve received } \\
44-60 \text { Gy ( } 56 \text { Gy); } \\
34 \% \text { when optic } \\
\text { nerve received } \\
61-78 \text { Gy at } 10 \text { yrs }\end{array}$ & 61-76 Gy: 24\%. \\
\hline $\begin{array}{l}\text { Logue } \\
{[6]^{\xi}}\end{array}$ & 152 & 61 & $\begin{array}{l}T_{4}: \\
44 \%\end{array}$ & $2 D$ & $\begin{array}{l}45-55 \\
\text { Gy/15-16 frx }\end{array}$ & $45.4 \%$ & $\mathrm{n} / \mathrm{a}$ & $\mathrm{n} / \mathrm{a}$ & $\begin{array}{l}9.9 \% \text { unilateral blindness, } \\
2.0 \% \text { bilateral blindness. }\end{array}$ \\
\hline \multirow{2}{*}{$\begin{array}{l}\text { Sakai } \\
{[7]^{\S}}\end{array}$} & \multirow[t]{2}{*}{171} & \multirow[t]{2}{*}{$\sim 60$} & \multirow[t]{2}{*}{$\mathrm{n} / \mathrm{a}$} & \multirow[t]{2}{*}{$2 D$} & \multirow{2}{*}{$\begin{array}{l}50-70 \\
\text { Gy/25-35 frx }\end{array}$} & \multirow[t]{2}{*}{$\mathrm{n} / \mathrm{a}$} & \multirow[t]{2}{*}{$\mathrm{n} / \mathrm{a}$} & |psilateral: 74.6\% & |psilateral: 63.7\% \\
\hline & & & & & & & & $\begin{array}{l}\text { Contralateral: } \\
36.8 \%\end{array}$ & Contralateral: $2.9 \%$ \\
\hline $\begin{array}{l}\text { Olmi } \\
{[8]}\end{array}$ & 69 & $\mathrm{n} / \mathrm{a}$ & $\begin{array}{l}\mathrm{T}_{3-4}: \\
88.4 \%\end{array}$ & $2 \mathrm{D}$ & $\begin{array}{l}42-72 \text { Gy, } 2 \\
\text { Gy daily or bid } \\
\text { delivered with } \\
\text { Co- } 60 \text { or electrons }\end{array}$ & $56 \%$ & $\mathrm{n} / \mathrm{a}$ & $\mathrm{n} / \mathrm{a}$ & $\begin{array}{l}13.6 \%(3 / 22) \text { blindness } \\
(60 \mathrm{~Gy} / 30 \mathrm{frx} \text { and } 52 \\
\text { Gy/26 bid frx })\end{array}$ \\
\hline $\begin{array}{l}\text { Nakissa } \\
{[9]}\end{array}$ & 30 & $\mathrm{n} / \mathrm{a}$ & $\mathrm{n} / \mathrm{a}$ & $2 \mathrm{D}$ & $\begin{array}{l}34-86 \text { Gy, various } \\
\text { fractionation }\end{array}$ & $\mathrm{n} / \mathrm{a}$ & $\mathrm{n} / \mathrm{a}$ & $\begin{array}{l}6.7 \% \text { (after } 65 \\
\text { Gy received) }\end{array}$ & $\begin{array}{l}6.7 \% \text { who received > } \\
68 \mathrm{~Gy}\end{array}$ \\
\hline
\end{tabular}

\# number of patients, $n / a$ not available, frx fraction, ${ }^{\dagger}$ postoperative only, ${ }^{*}$ associated with vision impairment or blindness, ON optic nerve, OC optic chiasm, ${ }^{*}$ median dose," average dose, pt patient, chemotherapy in a portion of pts".

received by the optic pathway $[5,9,11-16]$. The incidence of vision impairment appeared to be low when the optic pathway has received less than $60 \mathrm{~Gy}$. As shown by Jiang et al. the 10 year incidence of optic neuropathy was $2.3 \%$ when the optic nerve $(\mathrm{ON})$ received 44-60 Gy, which increased to $34 \%$ when the dose to the optic nerve was 61-78 Gy [5]. Optic chiasm injury, which led to bilateral blindness, increased from $8 \%$ to $24 \%$ when the OC dose increased from 50-60 Gy to 61-76 Gy [5]. In other studies, severe vision impairment/blindness was also more commonly found when radiation dose to the optic apparatus exceeded 60 Gy [9,13-15]. However, only 1 case of radiation related blindness was reported when the ON/OC dose was limited to $<54$ Gy $[11,12,16]$. Among all seventeen studies, a low incidence of blindness due to disease progression was observed in two studies that reported outcome after 3D RT alone and 2D/3D RT $[11,16]$. Both studies did not report any treatment related vision impairment.

Chemotherapy was combined with RT $(2.6 \%-100 \%$ of the patients in each study, mostly $<50 \%)$ predominantly in a sequential fashion in multiple studies [3,5-7,1012,14-18]. In these studies, the effect of chemotherapy on RT-related vision impairment has not been well described other than the fact concurrent chemo-radiation as reported by Homma et al. has been associated with a high incidence of severe optic toxicity [10].

\section{Local control \& optic toxicity after IMRT}

Among the 5 studies [22-26] which reported the clinical outcome \& toxicity profile after IMRT for sinonasal malignancies, no treatment related blindness was observed. Local control varied from $63.9 \%$ to $81 \%$ (Table 4). As shown in Table 4, no severe optic toxicity was reported when the dose to the ON/OC was kept to $\leq 54$ Gy (conventional fractionation). However, because of the short follow-up, and the relatively small number of patients, it remains to be seen whether 54 Gy is the dose limit for optimal visual preservation. Late severe vision impairment of $1.4 \%$ was observed when $<5 \%$ of the optic pathway was allowed to receive $\geq 60$ Gy [22]. Severe vision impairment due to tumor invasion is also 
Table 2 Optic toxicity after 3D RT for sinonasal malignancies

\begin{tabular}{|c|c|c|c|c|c|c|c|c|c|}
\hline Ref. & $\#$ & $\begin{array}{l}\text { Median follow } \\
\text { up (mo) }\end{array}$ & Stage & RT & Presciption dose & Local control & $\begin{array}{l}\text { Dose to the } \\
\text { optic pathway }\end{array}$ & Vision impairment & Blindness \\
\hline $\begin{array}{l}\mathrm{Homma} \\
{[10]^{\S}}\end{array}$ & 47 & 55.2 & $\begin{array}{l}\mathrm{T}_{4}: \\
85.1 \%\end{array}$ & $3 D$ & $\begin{array}{l}65 \text { Gy/26 frx or } \\
70 \text { Gy/35 frx }\end{array}$ & $83.0 \%$ & $\mathrm{n} / \mathrm{a}$ & $\begin{array}{l}42.1 \% \text { grade } 3 \text { or } 4 \\
\text { optic toxicity at } 2 \text { years } \\
\text { (CTCAE v.3); severe optic } \\
\text { toxicity is } 56 \% \text { in patients } \\
\text { with orbital invasion }\end{array}$ & None reported \\
\hline \multirow{4}{*}{$\begin{array}{l}\text { Gabriele } \\
{[11]^{\S}}\end{array}$} & \multirow[t]{4}{*}{31} & \multirow[t]{4}{*}{42} & \multirow{4}{*}{$\begin{array}{l}\mathrm{T}_{4}: \\
38.7 \%\end{array}$} & \multirow[t]{4}{*}{$3 \mathrm{D}$} & \multirow{4}{*}{$\begin{array}{l}\text { 60-68 } \\
\text { Gy/30-34 frx }\end{array}$} & $61.3 \%$ & ON: & \multirow[t]{4}{*}{ None } & \multirow{4}{*}{$\begin{array}{l}2 \text { due to disease } \\
\text { progression, } 1 \text { due } \\
\text { to cataract }\end{array}$} \\
\hline & & & & & & 5 yr LC: & $\begin{array}{l}\text {-Ipsilateral: } \\
\text { 5.7-46.1 Gy }\end{array}$ & & \\
\hline & & & & & & $\begin{array}{l}\text {-Postoperative: } \\
74 \%\end{array}$ & $\begin{array}{l}\text {-Contralateral: } \\
\text { 3.8-25.6 Gy }\end{array}$ & & \\
\hline & & & & & & $\begin{array}{l}\text {-Definitive: } \\
20 \%\end{array}$ & OC: 11.3-36.2 Gy & & \\
\hline \multirow[t]{4}{*}{$\begin{array}{l}\text { Pommier } \\
{[12]^{\S}}\end{array}$} & \multirow[t]{4}{*}{40} & \multirow[t]{4}{*}{19} & \multirow[t]{4}{*}{$\begin{array}{l}T_{3-4}: \\
55 \%\end{array}$} & \multirow[t]{4}{*}{$3 \mathrm{D}$} & $\begin{array}{l}\text { Definitive: } \\
63.5 \mathrm{~Gy}^{\natural}\end{array}$ & \multirow[t]{4}{*}{$80 \%$} & ON: & \multirow[t]{4}{*}{ None } & \multirow{4}{*}{$\begin{array}{l}1 \text { patient } 3 \text { years } \\
\text { after RT due to } \\
\text { vascular glaucoma }\end{array}$} \\
\hline & & & & & $\begin{array}{l}\text { Postoperative: } 61.8 \\
\text { - } 63.1 \mathrm{~Gy}^{\mathbf{4}}\end{array}$ & & $\begin{array}{l}\text {-Ipsilateral } \\
48.1 \mathrm{~Gy}^{\mathrm{A}}\end{array}$ & & \\
\hline & & & & & $2 \mathrm{~Gy} / \mathrm{frx}$ & & $\begin{array}{l}\text {-Contralateral } \\
22.0 \mathrm{~Gy}^{\mathrm{A}}\end{array}$ & & \\
\hline & & & & & & & OC: 43.5 Gy" & & \\
\hline \multirow[t]{3}{*}{ Roa [13] } & \multirow[t]{3}{*}{39} & \multirow[t]{3}{*}{54} & \multirow[t]{3}{*}{$\begin{array}{l}\text { IV: } \\
53.8 \%\end{array}$} & \multirow[t]{3}{*}{$3 \mathrm{D}$} & $\begin{array}{l}\text { Definitive: } \\
68.4 \mathrm{~Gy}^{\neq} ;\end{array}$ & \multirow[t]{3}{*}{$56.4 \%$} & $\mathrm{n} / \mathrm{a}$ & \multirow{3}{*}{$\begin{array}{l}1 \text { retinal artery occlusion } \\
\text { and } 1 \text { optic neuropathy } \\
\text { after } 68.4 \text { Gy delivered. }\end{array}$} & \multirow[t]{3}{*}{ None } \\
\hline & & & & & $\begin{array}{l}\text { Postoperative: } 55.8 \\
-67.8 \mathrm{~Gy}^{\ddagger}\end{array}$ & & & & \\
\hline & & & & & 1.8-2 Gy/frx & & & & \\
\hline
\end{tabular}

\# number of patients, $n / a$ not available, frx fraction, ${ }^{\dagger}$ postoperative, ${ }^{*}$ associated with vision impairment or blindness, ON optic nerve, OC optic chiasm, ${ }^{\ddagger}$ median dose, " average dose, pt patient, chemotherapy in a portion of pts.

reported by this group who treated the gross disease to 70 Gy. When compared with 3D or 2 D RT delivered at the same institution during an earlier era, three studies demonstrated noticeably improved clinical outcome and lower incidences of vision impairment and blindness after IMRT (Table 5).

Among IMRT-only studies, 16\% \& 56\% of the patients in studies by Hoppe et al. \& Wieger et al. respectively, have received chemotherapy as well $[25,26]$. In the IMRT vs. non-IMRT studies, $35.4 \%$ and $15 \%$ of the patients reported by Al-Mamgami et al. \& Chen et al. received chemotherapy $[28,29]$. No clear effect of chemotherapy on vision impairment after RT was observed in these studies.

Technique of IMRT delivery for the treatment of sinonasal malignancy

Among the 6 small studies [30-35] comparing IMRT and 2D/3D RT, IMRT was found to produce more conformal target volume coverage and/or improved sparing of optic structures when compared to non-IMRT techniques by some (Table 6). As shown by Claus et al. different IMRT strategies can lead to differences in PTV coverage and OAR sparing in the treatment of sinonasal malignancies [36]. Non-coplanar set up, increased number of beams
\& segments may lead to better target volume coverage and ON/OC sparing. Four studies further compared specific IMRT strategies [37-40]. Their findings are also described in Table 6. Although no differences between 5-beam coplanar \& non-coplanar IMRT in OAR sparing or target volume was shown by Serre et al. [38], partially non-coplanar IMRT has been shown to improve target volume dose coverage and sparing of optic structures when compared with 9-beam coplanar IMRT with Wang et al. [37]. An advanced technique of IMRT delivery under image guidance, helical tomotherapy (HT), was shown to be mostly comparable to 7-beam non-coplanar IMRT in ON/OC sparing, but superior in target dose homogeneity by Sheng et al. when a preoperative dose of 50 Gy was prescribed [39]. On the contrary, it was shown to generate both superior target dose homogeneity and optic structure sparing when compared to 9-11 beam coplanar IMRT in delivering 70 Gy/35 fractions by Chen et al. [40].

At last, the importance of incorporating clinical decision into the IMRT optimization process was demonstrated by Tsien et al. [41]. In their study to deliver $70 \mathrm{~Gy}$, the decision to spare only the contralateral optic pathway led to noticeably improved PTV coverage when compared to 3D CRT, which may result in better tumor 
Table 3 Optic toxicity after combined techniques for sinonasal malignancies

\begin{tabular}{|c|c|c|c|c|c|c|c|c|c|}
\hline Ref. & $\#$ & $\begin{array}{l}\text { Median } \\
\text { follow } \\
\text { up (mo) }\end{array}$ & Stage & RT & $\begin{array}{l}\text { Presciption } \\
\text { dose }\end{array}$ & Local control & Dose to the optic pathway & Vision impairment & Blindness \\
\hline \multirow[t]{4}{*}{ Jansen $[14]^{\S}$} & \multirow[t]{4}{*}{68} & \multirow[t]{4}{*}{66} & \multirow{4}{*}{$\begin{array}{l}T_{4}: \\
63.2 \%\end{array}$} & \multirow[t]{4}{*}{$2 \mathrm{D} / 3 \mathrm{D}$} & \multirow[t]{4}{*}{$66 \mathrm{~Gy} / 33 \mathrm{frx}{ }^{\neq}$} & $64.4 \%$ & LON: 73.8 & \multirow{4}{*}{$\begin{array}{l}27.9 \% ; 33.3 \% \text { patients } \\
\text { with orbital invasion } \\
\text { experienced severe } \\
\text { optic toxicity }\end{array}$} & \multirow[t]{4}{*}{$22.1 \%$} \\
\hline & & & & & & 5 yr LC: & $\mathrm{Gy}_{2}^{\neq^{*}}$ & & \\
\hline & & & & & & $\begin{array}{l}\text { Definitive: } \\
47 \%\end{array}$ & $\mathrm{RON}: 73.8 \mathrm{~Gy}_{2}^{\neq^{*}}$ & & \\
\hline & & & & & & $\begin{array}{l}\text { Postoperative: } \\
65 \%\end{array}$ & OC: $70 \mathrm{~Gy}_{2}^{\neq *}$ & & \\
\hline Snyers $[15]^{\S}$ & 168 & 69 & $\begin{array}{l}T_{4}: \\
57.6 \%\end{array}$ & $2 \mathrm{D} / 3 \mathrm{D}$ & 64 Gy/32 frx ${ }^{\neq}$ & $64 \%$ & $\begin{array}{l}1 \text { case of unilateral blindness } \\
\text { after both optic nerves } \\
\text { received a maximum dose of } \\
61 \mathrm{~Gy}\end{array}$ & $\begin{array}{l}14 \% \text { LENT SOMA } \\
\text { grade } 3-4 \\
\text { opthalmologic toxicity } \\
\text { at } 5 \text { years ( } 2 \text { D 15\%, 3D } \\
4 \% \text { ) }\end{array}$ & $\begin{array}{l}2.4 \% \\
\text { unilateral } \\
\text { blindness }\end{array}$ \\
\hline $\begin{array}{l}\text { Porceddu } \\
{[16]^{\S}}\end{array}$ & 60 & 67 & $\begin{array}{l}\mathrm{T}_{4}: \\
56.67 \%\end{array}$ & $2 \mathrm{D} / 3 \mathrm{D}$ & $\begin{array}{l}50-70 \text { Gy, } 1.8- \\
2 \text { Gy/frx; } \\
60 \text { Gy/30 frx } \\
\text { for 3D CRT }\end{array}$ & $62 \%$ & $\begin{array}{l}\text { ON/OC dose was limited to } \\
54 \text { Gy }\end{array}$ & $\mathrm{n} / \mathrm{a}$ & $\begin{array}{l}3 \text { due to } \\
\text { disease } \\
\text { progression }\end{array}$ \\
\hline \multirow[t]{4}{*}{ Blanco $[17]^{\S}$} & \multirow[t]{4}{*}{106} & \multirow[t]{4}{*}{60} & \multirow[t]{4}{*}{$\begin{array}{l}\mathrm{T}_{4}: \\
55.7 \%\end{array}$} & \multirow[t]{4}{*}{$2 \mathrm{D} / 3 \mathrm{D}$} & $\begin{array}{l}\text { Definitive } \\
61.7 \text { Gy }\end{array}$ & $58.5 \%$ & \multirow{4}{*}{$\begin{array}{l}1 \text { IMRT successfully spared } \\
\text { optic nerves and chiasm with } \\
\text { mean doses of } 33.9 \text { Gy \& } \\
38.9 \text { Gy to those two } \\
\text { structures }\end{array}$} & \multirow{4}{*}{$\begin{array}{l}\text { Optic neuropathy in } 2 \\
\text { pts }(1.9 \%) \& \text { optic } \\
\text { retinopathy in } 1 \mathrm{pt} \\
(0.9 \%) \text { all led to } \\
\text { blindness }\end{array}$} & \multirow[t]{4}{*}{$2.8 \%$} \\
\hline & & & & & $\begin{array}{l}\text { Postoperative } \\
60.9 \mathrm{~Gy}^{\natural}\end{array}$ & & & & \\
\hline & & & & & $\begin{array}{l}\text { Preoperative } \\
55.7 \mathrm{~Gy}^{9}\end{array}$ & & & & \\
\hline & & & & & $\begin{array}{l}\text { Mostly 1.8- } \\
2 \text { Gy/frx }\end{array}$ & & & & \\
\hline Hoppe $[18]^{\S}$ & 39 & 20 & $\begin{array}{l}\text { T4b: } \\
\text { 100\% }\end{array}$ & $\begin{array}{l}\text { 2D/ } \\
\text { 3D: } \\
69 \% \\
\text { IMRT } \\
31 \%\end{array}$ & $70 \mathrm{~Gy} / 35 \mathrm{frx}{ }^{\ddagger}$ & $35.9 \%$ & $\mathrm{n} / \mathrm{a}$ & $\begin{array}{l}1 \text { case of Optic } \\
\text { neuropathy, } 77 \text { Gy to } \\
\text { ON }\end{array}$ & $\begin{array}{l}1 \text { due to } \\
\text { optic } \\
\text { neuropathy } \\
7 \text { years after } \\
\text { IMRT }\end{array}$ \\
\hline \multirow[t]{6}{*}{$\begin{array}{l}\text { Mendenhall } \\
{[19]}\end{array}$} & \multirow[t]{6}{*}{109} & \multirow[t]{6}{*}{51.6} & \multirow[t]{6}{*}{$\begin{array}{l}\text { T4: } \\
32 \%\end{array}$} & \multirow[t]{6}{*}{$\begin{array}{l}2 \mathrm{D} / 3 \mathrm{D} \\
\text { mostly }\end{array}$} & $\begin{array}{l}\text { Preoperative: } \\
55 \mathrm{~Gy}^{\mp}\end{array}$ & 5 yr LC: $63 \%$ & \multirow[t]{6}{*}{$\mathrm{n} / \mathrm{a}$} & \multirow[t]{6}{*}{$\mathrm{n} / \mathrm{a}$} & $\begin{array}{l}\text { Definitive } \\
\text { RT: }\end{array}$ \\
\hline & & & & & $\begin{array}{l}\text { Postoperative: } \\
64.8 \mathrm{~Gy}^{\ddagger}\end{array}$ & T1-3: 82\% & & & $\begin{array}{l}\text {-Ipsilateral: } \\
25 \%\end{array}$ \\
\hline & & & & & & T4: $50 \%$ & & & $\begin{array}{l}\text {-Bilateral: } \\
1.8 \%\end{array}$ \\
\hline & & & & & $\begin{array}{l}\text { Definitive: } 70 \\
\text { Gy }^{\mp}\end{array}$ & $\begin{array}{l}\text { Definitive: } \\
43 \%\end{array}$ & & & $\begin{array}{l}\text { Pre or post } \\
\text { operative: }\end{array}$ \\
\hline & & & & & & $\begin{array}{l}\text { Postoperative: } \\
84 \%\end{array}$ & & & $\begin{array}{l}\text {-Ipsilateral: } \\
7.5 \%\end{array}$ \\
\hline & & & & & & & & & $\begin{array}{l}\text {-Bilateral: } \\
1.9 \%\end{array}$ \\
\hline
\end{tabular}

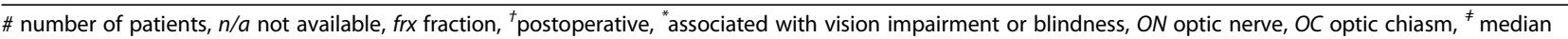
dose, " average dose, pt patient, chemotherapy in a portion of pts ${ }^{5}$.

control when compared to 3D CRT, and IMRT plans which were optimized to spare bilateral optic pathways.

\section{Discussion}

To our best knowledge, this is the first systematic review that investigates the clinical outcome and the level of optic pathway sparing after IMRT in comparison to 2D/3D RT for sinonasal malignancies. As described above, worse local control and high incidence of severe optic toxicity are mostly found after 2D RT (Tables 1, 2, 3 and 4). Improved local control and lower incidence of severe vision impairment was found after 3D CRT and IMRT, with IMRT being associated with the better local control and lower incidence of severe vision impairment in general (Tables 2, 4 and 5). As shown by Dirix et al. statistically significantly improved 2-year DFS and noticeably better toxicity profile was found following IMRT when compared with patients who were previous 
Table 4 Optic toxicity after IMRT for sinonasal malignancies

\begin{tabular}{|c|c|c|c|c|c|c|c|c|}
\hline Ref. & $\#$ & $\begin{array}{l}\text { Median } \\
\text { follow } \\
\text { up (mo) }\end{array}$ & Stage & Prescription dose & Local control & $\begin{array}{l}\text { Dose to the } \\
\text { optic pathway }\end{array}$ & Vision impairment & Blindness \\
\hline \multirow[t]{5}{*}{$\begin{array}{l}\text { Madani } \\
{[22]} \\
\text { CTCAE v2 }\end{array}$} & \multirow[t]{5}{*}{84} & \multirow[t]{5}{*}{40} & \multirow[t]{5}{*}{$\begin{array}{l}\text { T4: } \\
29 \%\end{array}$} & \multirow[t]{5}{*}{70 Gy/35 frx } & \multirow[t]{5}{*}{$77.4 \%$} & $\begin{array}{l}\text { ON/OC } \\
\text { constraint: } V_{60} \\
<5 \%\end{array}$ & Acute: $1.4 \%$ & \multirow[t]{5}{*}{ None } \\
\hline & & & & & & $\begin{array}{l}\text { Actual Dose to } \\
2 \% \text { vol": }\end{array}$ & \multirow{4}{*}{$\begin{array}{l}\text { Late:1.4\% Grade } 3 \text { visual impairment } \\
\text { related to IMRT in non-previously } \\
\text { treated pts; } 2.7 \% \text { grade } 3 \text { impairment } \\
\text { due to tumor invasion in both non- } \\
\text { previously irradiated \& re-irradiated pts }\end{array}$} & \\
\hline & & & & & & ON: & & \\
\hline & & & & & & $\begin{array}{l}\text { Ipsilateral } \\
58.4 \text { Gy; } \\
\text { Contralateral } \\
51.3 \text { Gy }\end{array}$ & & \\
\hline & & & & & & OC: $47.4 \mathrm{~Gy}$ & & \\
\hline \multirow[t]{4}{*}{$\begin{array}{l}\text { Combs } \\
{[23]}\end{array}$} & \multirow[t]{4}{*}{46} & \multirow[t]{4}{*}{16} & \multirow[t]{4}{*}{$\begin{array}{l}\text { T4: } \\
65 \%\end{array}$} & \multirow[t]{4}{*}{$\begin{array}{l}\text { PTV } 60 \text { Gy/30 frx, } \\
\text { bst CTV } 66 \text { Gy/33 frx }\end{array}$} & \multirow[t]{4}{*}{$81 \%$ at $2 \mathrm{yrs}$} & $\begin{array}{l}\text { ON/OC } \\
\text { constraint: } \\
54 \text { Gy. }\end{array}$ & \multirow[t]{4}{*}{ None } & \multirow[t]{4}{*}{ None } \\
\hline & & & & & & RON: $37.9 \mathrm{~Gy}^{\ddagger}$ & & \\
\hline & & & & & & LON: $37.4 \mathrm{~Gy}^{\ddagger}$ & & \\
\hline & & & & & & OC: $25.3 \mathrm{~Gy}^{\neq}$ & & \\
\hline \multirow[t]{6}{*}{ Daly [24] } & \multirow[t]{6}{*}{36} & \multirow[t]{6}{*}{51} & \multirow[t]{6}{*}{$\begin{array}{l}\text { T4: } \\
69 \%\end{array}$} & \multirow[t]{6}{*}{$\begin{array}{l}\text { GTV } 70 \text { Gy/33 } \\
\text { frx, CTV } 58 \text { Gy/33 frx }\end{array}$} & \multirow[t]{6}{*}{$63.9 \%$} & $\begin{array}{l}\text { ON/OC } \\
\text { constraints: } D_{1 \%} \\
54 \text { Gy (ON), } \\
45 \text { Gy (OC). }\end{array}$ & \multirow[t]{6}{*}{$\mathrm{N} / \mathrm{a}$} & \multirow[t]{6}{*}{ None } \\
\hline & & & & & & Actual $D_{\max }:$ & & \\
\hline & & & & & & OC: $52.3 \mathrm{~Gy}^{\mathrm{n}}$ & & \\
\hline & & & & & & ON: & & \\
\hline & & & & & & $\begin{array}{l}\text { Ipsilateral } \\
59.1 \mathrm{~Gy}^{\oplus}\end{array}$ & & \\
\hline & & & & & & $\begin{array}{l}\text { Contralateral } \\
45.2 \mathrm{~Gy}^{\sharp}\end{array}$ & & \\
\hline \multirow[t]{6}{*}{$\begin{array}{l}\text { Hoppe } \\
{[25]^{*}}\end{array}$} & \multirow[t]{6}{*}{37} & \multirow[t]{6}{*}{28} & \multirow[t]{6}{*}{$\begin{array}{l}\text { T4: } \\
55 \%\end{array}$} & PTV1 70 Gy & \multirow[t]{6}{*}{$72.9 \%$} & $\begin{array}{l}\text { ON/OC } \\
\text { constraint: < } \\
54 \text { Gy. }\end{array}$ & \multirow[t]{6}{*}{ No RTOG grade 3-4 toxicity } & \multirow[t]{6}{*}{ None } \\
\hline & & & & PTV2 60 Gy & & Actual $D_{\max }:$ & & \\
\hline & & & & PTV3 54 Gy & & ON & & \\
\hline & & & & \multirow[t]{3}{*}{ All in $33 \mathrm{frx}$} & & $\begin{array}{l}\text { ipsilateral } 53 \\
\mathrm{~Gy}^{\mp}\end{array}$ & & \\
\hline & & & & & & $\begin{array}{l}\text { contralateral } 41 \\
\mathrm{~Gy}^{\ddagger}\end{array}$ & & \\
\hline & & & & & & OC $50 \mathrm{~Gy}^{\neq}$ & & \\
\hline & 52 & 26.6 & T4: & High risk PTV 66 Gy/33 frx, & $75 \%$ & ON/OC & 1 grade 3 optic neuropathy related to & None \\
\hline CTCAE V3 & & & $16 \%$ & $\begin{array}{l}\text { 14.4 Gy, } 2 \text { Gy bid in } 5 \mathrm{pts} ; \\
\text { SRS/SRT bst in } 4 \text { pts: } 8 \text { Gy } x \\
1 \text { frx or } 5 \text { Gy } x 2 \mathrm{frx}\end{array}$ & $\begin{array}{l}\text { Postoperative: } \\
77 \%\end{array}$ & $\begin{array}{l}\text { constraint: } \\
45 \text { Gy, } 63 \text { Gy if } \\
\text { treated with bid }\end{array}$ & $\begin{array}{l}\text { herpes zoster infection, I grade } 3 \\
\text { corneal ulcer }\end{array}$ & \\
\hline & & & & & $\begin{array}{l}\text { Definitive: } \\
60 \%\end{array}$ & schedule. & & \\
\hline
\end{tabular}

\#: number of patients, *PTV1: residual disease, PTV2: surgical bed/areas at high risk for microscopic involvement, PTV3: elective nodal regions; $n / a$ not available; ${ }^{*}$ median dose; " average dose; ON optic nerve; OC optic chiasm; pts patients.

treated to the same doses with 3D CRT [27]. Although not statistically significant, improved local control in two other studies which compared IMRT and 3D and/or 2D RT were also seen, while one of them also demonstrated statistically significant improvement in optic organ preservation with IMRT over 3D CRT $[28,29]$. Further improvement in treatment outcome may lay in combining IMRT with newer class of systemic agents as those found in the treatment of other malignancies [42-45]. 
Table 5 Optic sparing, IMRT vs. non-IMRT

\begin{tabular}{|c|c|c|c|c|}
\hline Ref. & Dose & Clinical outcome & Dose to optic structures & Vision impairment \\
\hline \multirow[t]{3}{*}{ Dirix [27] } & \multirow[t]{3}{*}{$\begin{array}{l}\text { 60-66 Gy/30-33 frx for } \\
\text { both IMRT and 3D-CRT }\end{array}$} & $\begin{array}{l}\text { LC: } 76 \% \text { vs. } 67 \% \text { at } 2 \text { yrs } \\
\text { favoring IMRT }(p=0.06) \text {; }\end{array}$ & $\begin{array}{l}\text { ON/OC constraint: } \leq 60 \text { Gy for } \\
\text { IMRT. }\end{array}$ & No vision impairment after IMRT \\
\hline & & $\begin{array}{l}\text { DFS: } 72 \% \text { vs. } 60 \% \text { at } 2 \text { yrs } \\
\text { favoring IMRT }(p=0.02)\end{array}$ & & \multirow[t]{2}{*}{$\begin{array}{l}15.8 \% \text { radiation induced retinopathy after } \\
\text { 3D CRT. }\end{array}$} \\
\hline & & $\begin{array}{l}\text { OS: } 89 \% \text { vs. } 73 \% \text { at } 2 \text { yrs } \\
\text { favoring IMRT }(p=0.07)\end{array}$ & & \\
\hline \multirow{5}{*}{$\begin{array}{l}\text { Al- } \\
\text { Mamgani } \\
{[28]}\end{array}$} & IMRT(70\%): 60-74 Gy & \multirow[t]{5}{*}{$\begin{array}{l}\text { LC: } 80 \% \text { for IMRT \& } 64 \% \text { for } \\
\text { 3D CRT }(p=0.2)\end{array}$} & $\begin{array}{l}\operatorname{Dmax}^{\ddagger} \text { (IMRT vs. 3DCRT) when } \\
70 \text { Gy/35 frx was prescribed: }\end{array}$ & $\begin{array}{l}\text { Ocular toxicity: } 32 \% \text { after 3DCRT, } 5 \% \text { after } \\
\text { IMRT }(p<0.0001)\end{array}$ \\
\hline & \multirow[t]{4}{*}{ 3DCRT(30\%): 60-70 Gy } & & OC 47 Gy vs. 54 Gy & Blindness: 1 after IMRT, 3 after 3DCRT \\
\hline & & & ON & \multirow{3}{*}{$\begin{array}{l}\text { Organ preservation } 88 \% \text { vs. } 65 \% \text { favoring } \\
\text { IMRT }(p=0.01)\end{array}$} \\
\hline & & & -Ipsilateral 50 Gy vs. 56 Gy & \\
\hline & & & -Contralateral 42 Gy vs. 48 Gy & \\
\hline \multirow[t]{4}{*}{ Chen [29] } & 2D 50-74 Gy & LC: & $\mathrm{n} / \mathrm{a}$ & $\geq$ RTOG grade 3 visual toxicity \\
\hline & 3D 50-73 Gy & 2D: $55.9 \%$ & & $-2 \mathrm{D} 20 \%$ \\
\hline & \multirow[t]{2}{*}{ IMRT 66-72 Gy } & 3D: $67 \%$ & & $-3 D 9 \%$ \\
\hline & & IMRT: 69.6\% & & -IMRT 0\% \\
\hline
\end{tabular}

Blindness is only observed after 2D RT only (5.1\% of pts treated with 2D RT)

$\overline{O N}$ optic nerve, $O C$ optic chiasm; ${ }^{*}$ median dose; $n / a$ not available; $p t$ patient.

A few cases of blindness due to disease progression were reported in two studies $[11,16]$. No severe treatment related vision impairment was reported while a local control of approximately 60\% was achieved in both studies. This may be due to the difficulty of controlling locally very advanced disease. These studies included $38.7 \%$ and $56.67 \%$ of $\mathrm{T} 4$ disease, yet none had patients receiving more than 70 Gy $[11,16]$. The local control rate after definitive RT delivered with mostly non-IMRT techniques $[11,14,18,19]$ has been poor in general, especially for T4b disease even when a dose of 70 Gy was given [18]. Thus, a higher dose than previously used may need to be used when treating very locally advanced disease, with sacrificing the ipsilateral optic pathway being considered. On the other hand, superior conformal avoidance of the optic structures with IMRT may improve the outcome of definitive RT for sinonasal malignancies. This is evidenced by Wiegner et al. who observed a local control of only $60 \%$ after definitive IMRT delivering the conventionally accepted dose in a cohort of patients among whom $76 \%$ had T4 disease [26].

A high incidence of severe optic toxicity was observed following concurrent intra-arterial chemotherapy and 3D RT as shown by Homma et al. despite the excellent local control of $83 \%$ achieved in their study [10]. Thus, concurrent chemotherapy, such as intra-arterial cisplatin in this study, may potentially be a contributing factor to severe optic toxicity after radiotherapy for sinonasal malignancies. Such high rate of optic toxicity was not observed in other studies including patients who also received chemotherapy. Therefore, whether the addition of chemotherapy worsens optic toxicity following RT for sinonasal malignancies is unclear at this time.

IMRT can be challenging at times as no clear consensus exist on how to set the dose constraints for the optic structures (ON/OC). As previous described, a lower incidence of severe vision impairment has been reported in $2 \mathrm{D} / 3 \mathrm{D}$ studies when the radiation dose to the optic pathway was kept to $\leq 60$ Gy [3-19]. This finding was again evidenced in the IMRT studies. As an illustration, Madani et al. reported only $1.4 \%$ severe optic toxicity when $<5 \%$ of the optic pathway received more than 60 Gy [22]. The best chance of vision preservation and the avoidance of severe vision impairment appear to be associated with keeping the maximal radiation dose to the optic pathway to approximately 54 Gy or less [3-26]. This is also corroborated by the QUANTEC report on the radiation dose volume effect on the optic pathway [46]. However, it may be difficult to obtain if the tumor is close to the optic apparatus. Other confounding factors such as the volume of the optic apparatus receiving high radiation dose, the influence of chemotherapy and chronic conditions, such as, diabetes mellitus, warrants further investigation in the future [47]. Altered fractionation has not been shown to be associated with worse optic toxicity profiles when compared with other studies in which conventional fractionation is used $[5,6,8,9,25]$. This is especially true when the fractional dose was only slightly above 2 Gy [5,25]. pt]?>Thus, the dose volume effects of altered fractionation schedules with $>2$ Gy per fraction will need to be further characterized in the future since many studies were conducted in the pre-3D era 
Table 6 Techniques of IMRT delivery \pm comparison with 2D/3D techniques

\begin{tabular}{|c|c|c|c|c|}
\hline Ref. & Techniques & Dose prescribed & $\begin{array}{l}\text { ON/OC dose } \\
\text { constraints }\end{array}$ & Findings \\
\hline $\begin{array}{l}\text { Adams } \\
{[30]}\end{array}$ & IMRT vs. 2D/3D RT & $64 \mathrm{~Gy} / 32 \mathrm{frx}$ & $\begin{array}{l}\text { Contra-lateral } \\
\text { ON D } \max \leq \\
60 \mathrm{~Gy}\end{array}$ & $\begin{array}{l}\text { On average, IMRT decreased the } D_{\max } \text { to the contralateral ON } \\
\text { when compared with } 2 \mathrm{D} \& 3 \mathrm{D} \text { RT( } 56.4 \mathrm{~Gy} \text { vs. } 65.7 \mathrm{~Gy} \& 64.2 \mathrm{~Gy}) \text {, } \\
\text { and minimized volume receiving }<95 \% \text { prescribed dose ( } 8.5 \% \text { vs. } \\
15.1 \% \text {. } 14.7 \%)\end{array}$ \\
\hline Lee [31] & Static IMRT vs. 3D CRT & $70 \mathrm{~Gy} / 35 \mathrm{frx}$ & $D_{\max } \leq 60 \mathrm{~Gy}$ & $\begin{array}{l}\text { IMRT improved PTV coverage by the dose prescribed in general } \\
(93.0 \pm 2.2 \% \text { vs. } 89.0 \pm 4.8 \%, p=0.005) \text {, no benefit in OAR } \\
\text { sparing. }\end{array}$ \\
\hline $\begin{array}{l}\text { O'Daniel } \\
\text { [32] }\end{array}$ & Modulator IMRT vs. 3D CRT & 60-66 Gy/30-33 frx & $\mathrm{D}_{\max } \leq 54 \mathrm{~Gy}$ & $\begin{array}{l}35 \% \text { minimal transmission IMRT decreased Dmax to the ON/OC } \\
(p<0.05) \text {, and is comparable to static IMRT in ON/OC sparing }\end{array}$ \\
\hline $\begin{array}{l}\text { Huang } \\
{[33]}\end{array}$ & $\begin{array}{l}15 \text { beam IMRT; Sequential } \\
\text { tomotherapy (MIMic); 5-field 3D } \\
\text { CRT; 3-field 2D RT }\end{array}$ & $\begin{array}{l}\text { Minimal dose of } \\
60 \text { Gy to CTV \& } \\
70 \text { Gy to GTV }\end{array}$ & $\mathrm{D}_{\max } \leq 54 \mathrm{~Gy}$ & $\begin{array}{l}\text { IMRT achieved better GTV coverage \& sparing of OC when } \\
\text { compared to 2D \& 3D RT }\end{array}$ \\
\hline $\begin{array}{l}\text { Mock } \\
{[34]}\end{array}$ & $\begin{array}{l}\text { Passive scanning PT; IMRT; 3D } \\
\text { CRT; 2D RT }\end{array}$ & 60-70 Gy to the PTV & $\begin{array}{l}\leq 50 \text { Gy to } \\
\text { ON/OC }\end{array}$ & $\begin{array}{l}\text { Not significantly different, 3D CRT \& IMRT achieved better OAR } \\
\text { sparing than 2D RT. PT decreased OAR mean dose by }>60 \% \\
\text { when compared to 3D CRT \& IMRT. }\end{array}$ \\
\hline $\begin{array}{l}\text { Pacholke } \\
\text { [35] }\end{array}$ & $\begin{array}{l}6-7 \text { beam coplanar IMRT; } 3 \text { field; } \\
4 \text { field }\end{array}$ & $\begin{array}{l}70.2 \mathrm{~Gy} / 39 \mathrm{frx} \text { to } \\
\text { PTV }\end{array}$ & $\begin{array}{l}\text { Case } \\
\text { dependent }\end{array}$ & $\begin{array}{l}\text { IMRT did not demonstrate any clear advantage over } \\
\text { conventional } 4 \text { field plans. }\end{array}$ \\
\hline $\begin{array}{l}\text { Claus } \\
{[36]}\end{array}$ & $\begin{array}{l}\text { 4-11 beam IMRT, } 5 / 9 \text { beam } \\
\text { setups were coplanar }\end{array}$ & $70 \mathrm{~Gy} / 35 \mathrm{frx}$ & $\leq 60 \mathrm{~Gy}$ & $\begin{array}{l}\text { Increased beam \& segment number and non-coplanar setup } \\
\text { may lead to improved PTV dose coverage and improved OAR } \\
\text { sparing in selected cases. }\end{array}$ \\
\hline $\begin{array}{l}\text { Wang } \\
{[37]}\end{array}$ & $\begin{array}{l}9 \text { beam coplanar IMRT; } 5 \text { beam } \\
\text { non-coplanar/coplanar IMRT }\end{array}$ & 63 Gy/35 frx to PTV & $\mathrm{D}_{\max }<50 \mathrm{~Gy}$ & $\begin{array}{l}\text { Target dose better, mean dose to both ON \& } D_{\max } \text { to the } \\
\text { contralateral ON was significantly lower with the } 5 \text { beam } \\
\text { approach. }\end{array}$ \\
\hline Serre [38] & $\begin{array}{l}5 \text { beam coplanar IMRT; } 5 \text { beam } \\
\text { non-coplanar IMRT }\end{array}$ & $\mathrm{n} / \mathrm{a}$ & $D_{\max }<55$ Gy & $\begin{array}{l}\text { No obvious difference in OAR sparing \& target dose coverage } \\
\text { was found }\end{array}$ \\
\hline $\begin{array}{l}\text { Sheng } \\
{[39]}\end{array}$ & $\begin{array}{l}7 \text { beam non-coplanar IMRT vs. } \\
\text { HT }\end{array}$ & $50 \mathrm{~Gy} / 25 \mathrm{frx}$ & $\mathrm{n} / \mathrm{a}$ & $\begin{array}{l}\text { Comparable PTV dose coverage \& OAR sparing, although better } \\
\text { sparing of ipsilateral eye \& lens }\end{array}$ \\
\hline $\begin{array}{l}\text { Chen } \\
{[40]}\end{array}$ & 9-11 beam coplanar IMRT vs. HT & $70 \mathrm{~Gy} / 35 \mathrm{frx}$ & $D_{\max }: 54 \mathrm{~Gy}$ & $\begin{array}{l}\text { HT reduced Dmax to OC, ipsilateral ON \& retina; improved target } \\
\text { dose homogeneity }\end{array}$ \\
\hline Tsien [41] & $\begin{array}{l}9 \text { beam coplanar IMRT vs. 3D } \\
\text { CRT }\end{array}$ & $70 \mathrm{~Gy} / 35 \mathrm{frx}$ & $\mathrm{D}_{\max } \leq 60 \mathrm{~Gy}$ & $\begin{array}{l}\text { Clinical decision to spare the contralateral ON only can lead to } \\
\text { improved PTV dose coverage and improved TCP when } \\
\text { compared to bilateral ON sparing IMRT and 3D CRT. }\end{array}$ \\
\hline
\end{tabular}

ON optic nerve, OC optic chiasm, PTV planning target volume, OAR organs at risk, $P T$ proton therapy, $H T$ helical tomotherapy, frx fraction.

when dose to different structures cannot be accurately estimated.

Only small studies on a limited number of patients have conducted to compare IMRT with non-IMRT techniques, and various IMRT strategies [30-41]. Thus, no clear demonstration of the superiority of IMRT over 2D/ 3D techniques can be made based on these dosimetric studies. However, increased magnitude of intensity modulation through increasing the number of segments, beams, and using a combination of coplanar and noncoplanar arrangements may help increase dose conformality and optic pathway sparing when IMRT is used to treat sinonasal malignancies. This is suggested in studies which demonstrated HT's superiority over linac-based coplanar IMRT in target dose homogeneity and optic structure sparing; but comparable optic pathway sparing when HT and non-coplanar IMRT were compared $[39,40]$. HT's delivers image guided IMRT with intensity modulation through 51 equally spaced angles [48]. One important component of IMRT optimization to treat sinonasal malignancies is the incorporation of clinical decision into the optimization process. As shown by Tsien et al. decision to spare the contralateral ON only may lead to improved IMRT plan quality and potentially better tumor control in certain cases [41].

\section{Conclusion}

Dose conformality to the target volume and conformal avoidance of the organs at risk achieved through IMRT may provide better local control and less optic toxicity compared to conventional radiotherapy technique. A dose of 54 Gy or less delivered with conventional fractionation to the optic apparatus may provide optimal visual preservation if the tumor is not close to these critical structures. Further prospective studies with longterm follow-up should be conducted to assess how best to preserve vision and improve local control.

\section{Competing interests}

The authors declare that they have no competing interests. 


\section{Authors' contribution}

$A C$ and $A Z$ originated the idea and $A C$ and NP conducted the design of this review. $A C, N P, W T, G S, C P$, and $A Z$ conducted the data gathering and the writing of this manuscript. All authors approved this final version.

\section{Author details}

${ }^{1}$ Department of Radiation Oncology, West Virginia University, 1 Medical Center Dr. Morgantown, Morgantown, WV 26506, USA. ${ }^{2}$ Department of Radiation Oncology, University of Arizona, Tucson, AZ 85724, USA. ${ }^{3}$ Department of Hematology Oncology, West Virginia University, Morgantown, WV 26506, USA. ${ }^{4}$ Department of Radiation Oncology, Michael E. Debakey VA Medical Center, Houston, TX 77030, USA

Received: 1 October 2012 Accepted: 31 December 2012

Published: 7 January 2013

\section{References}

1. Dulguerov P, Jacobson MS, Allal AS, et al: Nasal and paranasal sinus carcinoma: are we making progress? A series of 220 patients and a systematic review. Cancer 2001, 92:3012-3029.

2. Ang KK, Ahamad A, Garden AS: The nasal cavity and paranasal sinuses. In Radiation Oncology: Rationale, Technique, Results. 9th edition. Edited by Cox JD, Ang KK. Philadelphia: Mosby, Inc; 2010:183-206.

3. Ogawa K, Toita T, Kakinohana Y, et al: Postoperative radiotherapy for squamous cell carcinoma of the maxillary sinus: analysis of local control and late complications. Oncol Rep 2001, 8:315-319.

4. Tiwari R, Hardillo JA, Tobi $H$, et al: Carcinoma of the ethmoid: results $f$ treatment with conventional surgery and post-operative radiotherapy. Eur J Surg Oncol 1999, 25:401-405.

5. Jiang GL, Tucker SL, Guttenberger R, et al: Radiation-induced injury to the visual pathway. Radiother Oncol 1994, 30:17-25.

6. Logue JP, Slevin NJ: Carcinoma of the nasal cavity and paranasal sinuses: an analysis of radical radiotherapy. Clin Oncol 1991, 3:84-89.

7. Sakai S, Kubo T, Mori N, et al: A study of the late effects of radiotherapy and operation on patients with maxillary cancer. A survey more than 10 years after initial treatment. Cancer 1988, 62:2114-2117.

8. Olmi P, Cellai E, Chiavacci A, et al: Paranasal sinuses and nasal cavity cancer: different radiotherapeutic options, results and late damages. Tumori 1986, 72:589-595.

9. Nakissa N, Rubin P, Strohl, et al: Ocular and orbital complications following radiation therapy of paranasal sinus malignancies and review of literature. Cancer 1983, 51:980-986.

10. Homma A, Oridate N, Suzuki F, et al: Superselective high-dose cisplatin with concomitant radiotherapy in patients with advanced cancer of the nasal cavity and paranasal sinuses: a single institution experience. Cancer 2009, 115:4705-4714.

11. Gabriele AM, Airoldi M, Garzaro M, et al: Stage III-IV sinonasal and nasal cavity carcinoma treated with three-dimensional conformal radiotherapy. Tumori 2008, 94:320-326.

12. Pommier $P$, Ginestet $C$, Sunyach MP, et al: Conformal radiotherapy for paranasal sinus and nasal cavity tumors: three-dimensional treatment planning and preliminary results in 40 patients. Int I Radiat Oncol Biol Phys 2000, 48:485-493.

13. Roa WH, Hazuka MB, Sandler HM, et al: Results of primary and adjuvant CT-based 3-dimensional radiotherapy for malignant tumors of the paranasal sinuses. Int J Radiat Oncol Biol Phys 1994, 28:857-865.

14. Jansen EP, Keus RB, Hilgers FJ, et al: Does the combination of radiotherapy and debulking surgery favor survival in paranasal sinus carcinoma? Int J Radiat Oncol Biol Phys 2000, 48:27-35.

15. Snyers A, Janssens GO, Twickler MB, et al: Malignant tumors of the nasal cavity and paranasal sinuses: long-term outcome and morbidity with emphasis on hypothalamic-pituitary deficiency. Int J Radiat Oncol Biol Phys 2009, 73:1343-1351.

16. Porceddu S, Martin J, Shanker G, et al: Paranasal sinus tumors: peter maccallum cancer institute experience. Head Neck 2004, 26:322-330.

17. Blanco Al, Chao KS, Ozyigit G, et al: Carcinoma of paranasal sinuses: longterm outcomes with radiotherapy. Int J Radiat Oncol Biol Phys 2004, 59:51-58.

18. Hoppe BS, Nelson CJ, Gomez DR, et al: Unresectable carcinoma of the paranasal sinuses: outcomes and toxicities. Int I Radiat Oncol Biol Phys 2008, 72:763-769.
19. Mendenhall WM, Amdur RJ, Morris CG, et al: Carcinoma of the nasal cavity and paranasal sinuses. Laryngoscope 2009, 119:899-906.

20. Claus F, De Gersem W, De Wagter C, et al: An implementation strategy for IMRT of ethmoid sinus cancer with bilateral sparing of the optic pathways. Int I Radiat Oncol Biol Phys 2001, 51:318-331.

21. Duthoy W, Boterberg T, Claus F, et al: Postoperative intensity-modulated radiotherapy in sinonasal carcinoma: clinical results in 39 patients. Cancer 2005, 104:71-82.

22. Madani I, Bonte K, Vakaet $L$, et al: Intensity-modulated radiotherapy for sinonasal tumors: Ghent University Hospital update. Int J Radiat Oncol Biol Phys 2009, 73:424-432.

23. Combs SE, Konkel S, Schulz-Ertner D, et al: Intensity modulated radiotherapy (IMRT) in patients with carcinomas of the paranasal sinuses: clinical benefit for complex shaped target volumes. Radiat Oncol 2006, 1:23.

24. Daly ME, Chen AM, Kara Bucci M, et al: Intensity-modulated radiation therapy for malignancies of the nasal cavity and paranasal sinuses. Int J Radiat Oncol Biol Phys 2007, 67:151-157.

25. Hoppe BS, Wolden SL, Zelefsky MJ, et al: Postoperative intensitymodulated radiation therapy for cancers of the paranasal sinuses, nasal cavity, and lacrimal glands: technique, early outcomes, and toxicity. Head Neck 2008, 30:925-932.

26. Wiegner EA, Daly ME, Murphy JD, et al: Intensity-modulated radiotherapy for tumors of the nasal cavity and paranasal sinuses: Clinical outcomes and patterns of failure. Int J Radiat Oncol Biol Phys 2012, 83:243-251.

27. Dirix $P$, Vanstraelen $B$, Jorissen $M$, et al: Intensity-modulated radiotherapy for sinonasal cancer: improved outcome compared to conventional radiotherapy. Int J Radiat Oncol Biol Phys 2010, 78:998-1004.

28. Al-Mamgani A, Monserez D, Rooij PV, et al: Highly-conformal intensitymodulated radiotherapy reduced toxicity without jeopardizing outcome in patients with paranasal sinus cancer treated by surgery and radiotherapy or (chemo) radiation. Oral Oncol 2012, In press.

29. Chen AM, Daly ME, Kara Bucci M, et al: Carcinomas of the paranasal sinuses and nasal cavity treated with radiotherapy at a single institution over five decades: are we making improvement? Int I Radiat Oncol Biol Phys 2007, 69:141-147.

30. Adams EJ, Nutting CM, Convery DJ, et al: Potential role of intensitymodulated radiotherapy in the treatment of tumors of the maxillary sinus. Int J Radiat Oncol Biol Phys 2001, 51:579-588.

31. Lee SW, Kim GE, Suh CO, et al: Intensity modulation technique using the complementary boost-fields for ethmoid sinus cancer. Clin Oncol 2002, 14:241-249.

32. O'Daniel JC, Dong L, Kuban DA, et al: The delivery of IMRT with a single physical modulator for multiple fields: a feasibility study for paranasal sinus cancer. Int J Radiat Oncol Biol Phys 2004, 58:876-887.

33. Huang $D, X i a P$, Akazawa $P$, et al: Comparison of treatment plans using intensity-modulated radiotherapy and three-dimensional conformal radiotherapy for paranasal sinus carcinoma. Int J Radiat Oncol Biol Phys 2003, 56:158-168.

34. Mock U, Georg D, Bogner J, et al: Treatment planning comparison of conventional, 3D conformal, and intensity-modulated photon (IMRT) and proton therapy for paranasal sinus carcinoma. Int I Radiat Oncol Biol Phys 2004, 58:147-154.

35. Pacholke HD, Amdur RJ, Louis DA, et al: The role of intensity modulated radiation therapy for favorable stage tumor of the nasal cavity or ethmoid sinus. Am J Clin Oncol 2005, 28:474-478.

36. Claus F, Mijnheer B, Rasch C, et al: Report of a study on IMRT planning strategies for ethmoid sinus cancer. Strahlenther Onkol 2002, 178:572-576.

37. Wang $X$, Zhang $X$, Dong $L$, et al: Effectiveness of noncoplanar IMRT planning using a parallelized multiresolution beam angle optimization method for paranasal sinus carcinoma. Int J Radiat Oncol Biol Phys 2005, 63:594-601.

38. Serre A, Idri K, Fenoglietto P, et al: Dosimetric comparison between coplanar and non coplanar field radiotherapy for ethmoid sinus cancer. Radiat Oncol 2007, 2:35.

39. Sheng K, Molloy JA, Larner JM, et al: A dosimetric comparison of noncoplanar IMRT versus helical tomotherapy for nasal cavity and paranasal sinus cancer. Radiother Oncol 2007, 82:174-178.

40. Chen AM, Sreeraman R, Mathai M, Vijayakumar S, Purdy JA: Potential of helical tomotherapy to reduce dose to the ocular structures for patients treated for unresectable sinonasal cancer. Am J Clin Oncol 2010, 33:595-598. 
41. Tsien C, Eisbruch A, McShan D, et al: Intensity-modulated radiation therapy (IMRT) for locally advanced paranasal sinus tumors: incorporating clinical decisions in the optimization process. Int J Radiat Oncol Biol Phys 2003, 55:776-784.

42. Elbaz H, Stueckle T, Tse W, et al: Digitoxin and its analogs as novel cancer therapeutics. Ex Hematol Oncol 2012, 1:4.

43. Weiß L, Efferth T: Polo-like kinase 1 as target for cancer therapy. Exp Hematol Oncol 2012, 1:38.

44. Lamba G, Gupta R, Lee B, et al: Current management and prognostic features for gastrointestinal stromal tumor (GIST). Exp Hematol Oncol 2012, 1:14.

45. Rafiyath $S M$, Rasul M, Lee B, et al: Comparison of safety and toxicity of liposomal doxorubicin vs. conventional anthracyclines; a meta-analysis. Exp Hematol Oncol 2012, 1:10.

46. Mayo C, Martel MK, Marks LB, et al: Radiation dose-volume effects of optic nerves and chiasm. Int J Radiat Oncol Biol Phys 2010, 76(3 Suppl):S28-S35.

47. Martel MK, Sandler HM, Cornblath WT, et al: Dose-volume complication analysis for visual pathway structures of patients with advanced paranasal sinus tumors. Int J Radiat Oncol Biol Phys 1997, 38:273-284.

48. Chi A, Jang SY, Welsh JS, et al: Feasibility of helical tomotherapy in stereotactic body radiation therapy for centrally located early stage nonsmall-cell lung cancer or lung metastases. Int J Radiat Oncol Biol Phys 2011, 81:856-862.

doi:10.1186/1756-8722-6-4

Cite this article as: Chi et al:: Intensity modulated radiotherapy for sinonasal malignancies with a focus on optic pathway preservation. Journal of Hematology \& Oncology 2013 6:4.

\section{Submit your next manuscript to BioMed Central and take full advantage of:}

- Convenient online submission

- Thorough peer review

- No space constraints or color figure charges

- Immediate publication on acceptance

- Inclusion in PubMed, CAS, Scopus and Google Scholar

- Research which is freely available for redistribution 